Case Report

\title{
Novel Mutation in a Patient with Cholesterol Ester Storage Disease
}

\author{
Patrick Lin,, ${ }^{1,2}$ Sheela Raikar, ${ }^{1,2,3}$ Jennifer Jimenez, ${ }^{1,2,3}$ \\ Katrina Conard, ${ }^{4}$ and Katryn N. Furuya ${ }^{1,2,3}$ \\ ${ }^{1}$ Department of Pediatrics, Nemours/Alfred I. duPont Hospital for Children, Wilmington, DE 19803, USA \\ ${ }^{2}$ Thomas Jefferson University, Philadelphia, PA 19107, USA \\ ${ }^{3}$ Division of Pediatric Gastroenterology, Hepatology, and Nutrition, Nemours/Alfred I. duPont Hospital for Children, \\ Wilmington, DE 19803, USA \\ ${ }^{4}$ Department of Clinical and Anatomic Pathology, Nemours/Alfred I. duPont Hospital for Children, Wilmington, DE 19803, USA
}

Correspondence should be addressed to Katryn N. Furuya; kfuruya@nemours.org

Received 21 November 2014; Accepted 27 January 2015

Academic Editor: Yoshiyuki Ban

Copyright (C) 2015 Patrick Lin et al. This is an open access article distributed under the Creative Commons Attribution License, which permits unrestricted use, distribution, and reproduction in any medium, provided the original work is properly cited.

Cholesterol ester storage disease (CESD) is a chronic liver disease that typically presents with hepatomegaly. It is characterized by hypercholesterolemia, hypertriglyceridemia, high-density lipoprotein deficiency, and abnormal lipid deposition within multiple organs. It is an autosomal recessive disease that is due to a deficiency in lysosomal acid lipase (LAL) activity, which is coded by the lysosomal acid lipase gene (LIPA). We describe the case of a 5-year-old south Asian female incidentally found to have hepatomegaly, and subsequent workup confirmed the diagnosis of CESD. DNA sequencing confirmed the presence of a novel hepatic mutation. It is a four-nucleotide deletion c.57_60delTGAG in exon 2 of the LIPA gene. This mutation is predicted to result in a premature translation stop downstream of the deletion (p.E20fs) and, therefore, is felt to be a disease-causing mutation.

\section{Introduction}

Cholesterol ester storage disease (CESD) is a chronic liver disease that typically presents with hepatomegaly. It is associated with hypercholesterolemia, hypertriglyceridemia, highdensity lipoprotein (HDL) deficiency, and abnormal lipid deposition within multiple organs [1]. It is an autosomal recessive disease that is due to a deficiency in lysosomal acid lipase (LAL) activity. LAL hydrolyzes cholesterol esters and triglycerides within the lysosomes of hepatocytes. In the absence of LAL, lipid builds up in the endoplasmic reticulum of hepatocytes. This leads to the development of hepatic steatosis with the eventual development of fibrosis and micronodular cirrhosis $[2,3]$.

Disease-causing mutations in the LAL gene (lysosomal acid lipase gene [LIPA]) may result in the clinical presentation of CESD or Wolman disease (WD). Wolman disease is a severe, early-onset presentation caused by a mutation in the LIPA gene that results in the absence of LAL activity [2]. It is typically fatal within the first few months of life. On the other hand, CESD is more prevalent than WD and typically presents later in life. We present a patient with CESD who has a novel disease-causing mutation in the LIPA gene.

\section{Case Presentation}

A 5-year-old south Asian female was incidentally found to have hepatomegaly on a trip to India, where she became acutely ill with fever, vomiting, and abdominal pain. Blood work done at that time demonstrated mildly elevated liver function tests (AST $48 \mathrm{U} / \mathrm{L}$, ALT $80 \mathrm{U} / \mathrm{L}$ ), and an abdominal ultrasound revealed hepatomegaly without biliary or splenic abnormalities. She was prescribed an antibiotic and her symptoms gradually resolved.

A month after her return to the United States, her pediatrician noted persistent hepatomegaly and the new development of fever. She was referred to the emergency department because of suspicion of a tropical infectious 


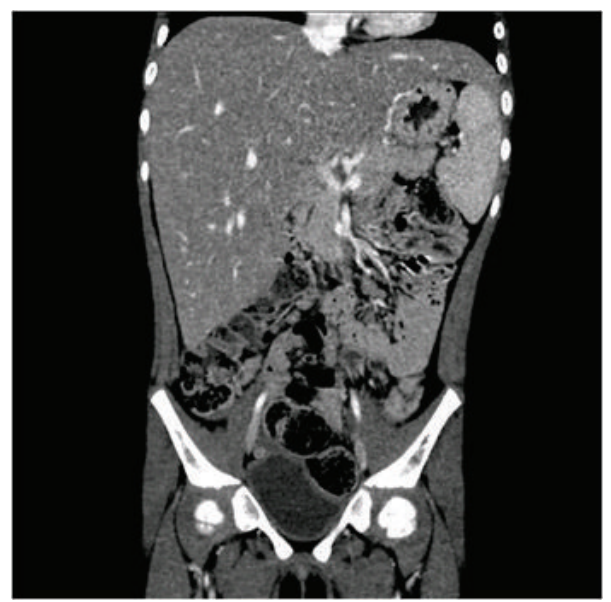

FIGURE 1: Computed tomography of abdomen and pelvis. The liver is enlarged, measuring up to $17 \mathrm{~cm}$ in width and craniocaudal dimension. The contour is smooth. The hepatic parenchyma is homogenous without a focal mass. There is no intrahepatic biliary dilatation. The portal and hepatic venous systems are patent and nondilated.

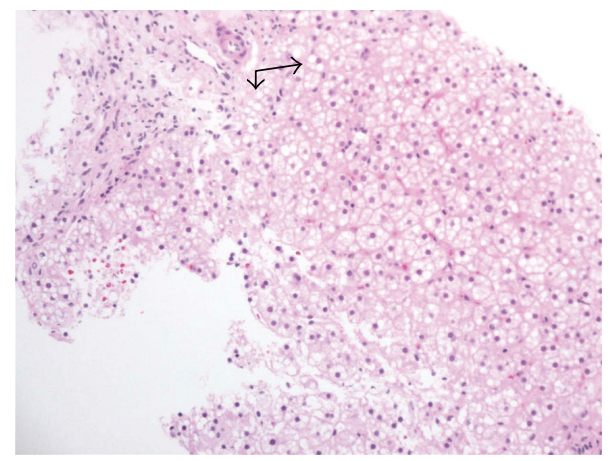

(a)

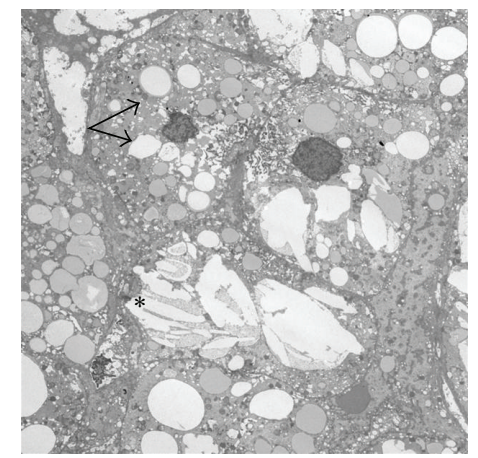

(b)

Figure 2: (a) Liver biopsy, periodic acid-Schiff stain with diastase (magnification, $\times 40$ ). Lipid demonstrated within the hepatocellular cytoplasm (arrows). (b) Electron micrograph (direct magnification, $\times 2500$ ). The hepatocellular cytoplasm in places has a moth-eaten appearance with lipid droplets (arrows). Additionally, some foci show cholesterol crystals $(*)$ free in the hepatocellular cytoplasm.

disease. A repeat ultrasound demonstrated persistent hepatomegaly. Her transaminases (AST $133 \mathrm{U} / \mathrm{L}$, ALT $112 \mathrm{U} / \mathrm{L}$ ) remained elevated. The infectious workup was negative for cytomegalovirus, hepatitis A, Lyme disease, and malaria. She did have a positive Epstein-Barr virus IgM. Repeat blood work was obtained at follow-up infectious disease clinic visit where she was again found to have hepatomegaly $(7 \mathrm{~cm}$ below the costal margin) and mildly abnormal transaminases. A computed tomography of the abdomen and pelvis (Figure 1) was performed that demonstrated hepatomegaly but had otherwise normal findings. Her fever resolved within 2 weeks. She was otherwise well and was not on any medications. There was no family history of liver disease. Her immunizations were up to date. Because of persistent hepatomegaly, she was referred to the liver clinic.

In the liver clinic, she was noted to have an enlarged, firm liver, palpable $8 \mathrm{~cm}$ below the costal margin with no associated splenomegaly. Test results for autoimmune hepatitis, WD, and alpha-1-antitrypsin deficiency were negative. Nonfasting lipid levels were abnormal; repeat testing showed a fasting total cholesterol of $305 \mathrm{mg} / \mathrm{dL}$ and a triglyceride level of $142 \mathrm{mg} / \mathrm{dL}$.

On the basis of a clinical suspicion of a diagnosis of CESD, a liver biopsy was performed. It revealed diffuse, microvesicular steatosis in the hepatic parenchyma (Figure 2(a)). Portal tracts were expanded by foamy macrophages containing finely vacuolated material, which was periodic acid-Schiff positive and resistant to diastase digestion. Trichrome stain for collagen showed mild portal fibrosis with early delicate bridging fibrosis. Ultrastructurally, the cytoplasm had a moth-eaten appearance with the presence of lipid droplets and cholesterol crystals (Figure 2(b)). There was no evidence of hepatocellular necrosis, cholestasis, or bile duct proliferation.

Liver tissue was sent to Dr. D. Wenger's Lysosomal Diseases Testing Laboratory at Thomas Jefferson University. She was found to have a low acid lipase activity of 7.2 (no units provided by Dr. D. Wenger's laboratory) in the liver. Follow-up testing through a commercial laboratory demonstrated low level of lysosomal acid lipase 
activity $(0.008 \mathrm{nmol} / \mathrm{punch} / \mathrm{h})$ in blood. Lysosomal acid lipase gene sequence analysis was performed, which demonstrated that she was heterozygous for the following two mutations: c.57_60delTGAG and c.894G > A. Both parents underwent mutation analysis. The mother was confirmed to have the c.894G $>$ A mutation while the father carried the c.57_60delTGAG mutation. The c.57_60delTGAG is a new mutation not previously described in the literature in patients with CESD.

\section{Discussion}

Cholesterol ester storage disease is an autosomal recessive, chronic liver disease caused by LAL deficiency. Its cognate gene is located on chromosome 10q23.3-q23.3 [2]. Lysosomal acid lipase hydrolyzes cholesterol esters and triglycerides that are delivered to the lysosomes by receptor-mediated endocytosis, and deficiency states result in accumulation of both cholesterol esters and triglycerides in hepatocytes [2]. Mutations in this gene cause two distinct phenotypes: WD and CESD. Both are characterized by hypercholesterolemia, hypertriglyceridemia, HDL deficiency, and hepatomegaly secondary to hepatic steatosis [2]. Complete absence of LAL activity results in $\mathrm{WD}$, whereas CESD is due to mutations that result in partial loss of enzyme activity. Children diagnosed with CESD generally have a better prognosis but may often still require liver transplantation during their lifetime. They are also at risk for developing complications from cardiac disease.

Our patient's symptoms and presentation are typical of CESD. The histologic and ultrastructural examination of the liver along with markedly low LAL activity confirmed this diagnosis. DNA sequencing confirmed the presence of a novel hepatic mutation. It is a four-nucleotide deletion, c.57_60delTGAG in exon 2 of the LIPA gene. This mutation is predicted to result in a premature translation stop downstream of the deletion (p.E20fs) and, therefore, is felt to be a disease-causing mutation [4]. The second mutation noted in our patient is a previously described disease-causing mutation, c.894G $>$ A change in exon 8 of the LIPA gene, which results in altered mRNA splicing and exon 8 skipping [5].

Current management is limited to preventing adverse effects of dyslipidemia. The use of statins $[6,7]$ and other cholesterol lowering agents such as cholestyramine and ezetimibe $[1,8,9]$ has been associated with decreasing total cholesterol and increasing HDL, which is cardioprotective. Due to the young age of our patient, neither statins nor ezetimibe were prescribed for her hypercholesterolemia. In addition, lipid accumulation in the liver is not reversed with these medications. Patients may go on to develop cirrhosis and liver failure. Liver transplantation as a therapeutic option has been successfully used $[1,10,11]$. Direct enzyme replacement therapy with sebelipase alfa has been recently developed by Synageva BioPharma (Lexington, MA, USA) and is currently undergoing human trials $[3,12]$. It has been recently reported in a phase 3 double blind placebo controlled trial that sebelipase alfa replacement enzyme therapy resulted in an improvement in ALT and AST with a relative reduction in hepatic fat fraction [13]. Thus, enzyme replacement therapy is a promising therapy that may change the long term outcome for patients with WD and CESD.

\section{Disclosure}

Patrick Lin and Sheela Raikar are co-first authors.

\section{Conflict of Interests}

The authors declare that there is no conflict of interests regarding the publication of this paper.

\section{References}

[1] D. L. Bernstein, H. Hülkova, M. G. Bialer, and R. J. Desnick, "Cholesteryl ester storage disease: review of the findings in 135 reported patients with an underdiagnosed disease," Journal of Hepatology, vol. 58, no. 6, pp. 1230-1243, 2013.

[2] S. Muntoni, H. Wiebusch, M. Jansen-Rust et al., "Prevalence of cholesteryl ester storage disease," Arteriosclerosis, Thrombosis, and Vascular Biology, vol. 27, no. 8, pp. 1866-1868, 2007.

[3] T. Reynolds, "Cholesteryl ester storage disease: a rare and possibly treatable cause of premature vascular disease and cirrhosis," Journal of Clinical Pathology, vol. 66, no. 11, pp. 918923, 2013.

[4] C. S. Richards, S. Bale, D. B. Bellissimo et al., "ACMG recommendations for standards for interpretation and reporting of sequence variations: revisions 2007," Genetics in Medicine, vol. 10, no. 4, pp. 294-300, 2008.

[5] H. Klima, K. Ullrich, C. Aslanidis, P. Fehringer, K. J. Lackner, and G. Schmitz, "A splice junction mutation causes deletion of a 72-base exon from the mRNA for lysosomal acid lipase in a patient with cholesteryl ester storage disease," The Journal of Clinical Investigation, vol. 92, no. 6, pp. 2713-2718, 1993.

[6] B. Dalgiç, S. Sari, M. Gündüz et al., "Cholesteryl ester storage disease in a young child presenting as isolated hepatomegaly treated with simvastatin," The Turkish Journal of Pediatrics, vol. 48, no. 2, pp. 148-151, 2006.

[7] S. A. Iverson, S. R. Cairns, C. P. Ward, and A. H. Fensom, "Asymptomatic cholesteryl ester storage disease in an adult controlled with simvastatin," Annals of Clinical Biochemistry, vol. 34, no. 4, pp. 433-436, 1997.

[8] L. Leone, P. F. Ippoliti, and R. Antonicelli, "Use of simvastatin plus cholestyramine in the treatment of lysosomal acid lipase deficiency," The Journal of Pediatrics, vol. 119, no. 6, pp. 10081009, 1991.

[9] V. T. Tadiboyina, D. M. Liu, B. A. Miskie, J. Wang, and R. A. Hegele, "Treatment of dyslipidemia with lovastatin and ezetimibe in an adolescent with cholesterol ester storage disease," Lipids in Health and Disease, vol. 4, article 26, 2005.

[10] J. N. Arterburn, W. M. Lee, R. P. Wood, B. W. Shaw, and R. S. Markin, "Orthotopic liver transplantation for cholesteryl ester storage disease," Journal of Clinical Gastroenterology, vol. 13, no. 4, pp. 482-484, 1991.

[11] G. D. Ferry, H. H. Whisennand, M. J. Finegold, E. Alpert, and A. Glombicki, "Liver transplantation for cholesteryl ester storage disease," Journal of Pediatric Gastroenterology and Nutrition, vol. 12, no. 3, pp. 376-378, 1991. 
[12] H. Du, T. L. Cameron, S. J. Garger et al., "Wolman disease/cholesteryl ester storage disease: efficacy of plant-produced human lysosomal acid lipase in mice," The Journal of Lipid Research, vol. 49, no. 8, pp. 1646-1657, 2008.

[13] M. Balwani, B. Burton, I. Baric et al., "Results of a global phase 3, randomized, double-blind, placebo-controlled trial evaluation the efficacy and therapy in children and adults with lysosomal acid lipase deficiency," Hepatology, vol. 60, no. 6, p. 127, 2014. 


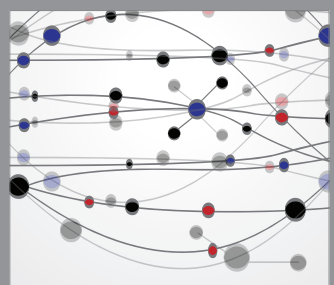

The Scientific World Journal
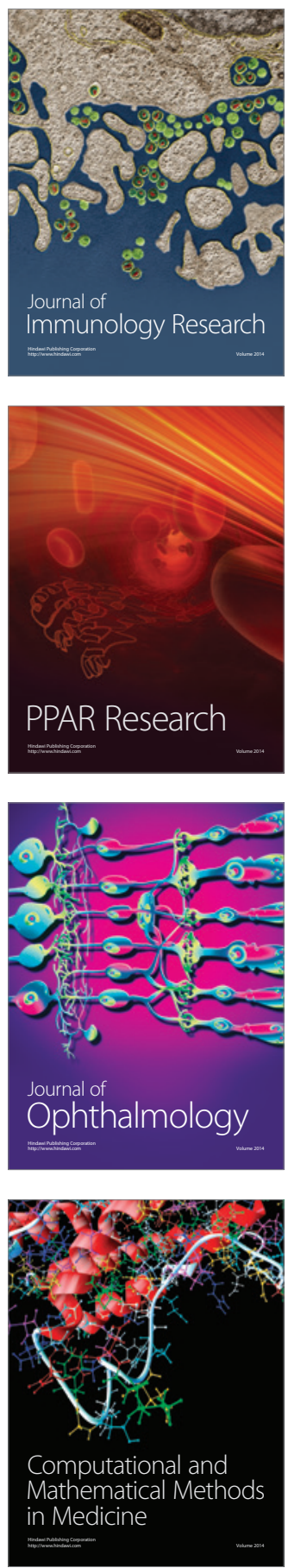

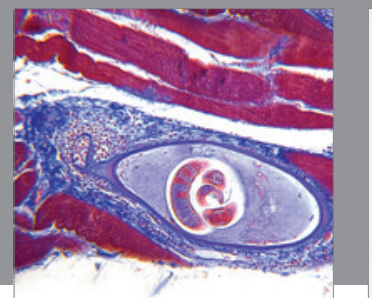

Gastroenterology

Research and Practice
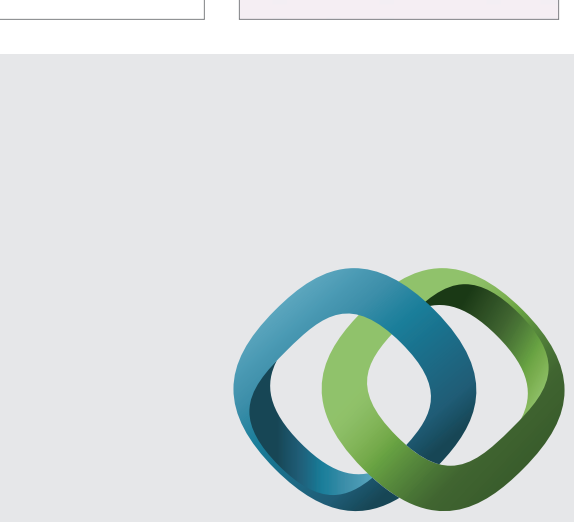

\section{Hindawi}

Submit your manuscripts at

http://www.hindawi.com
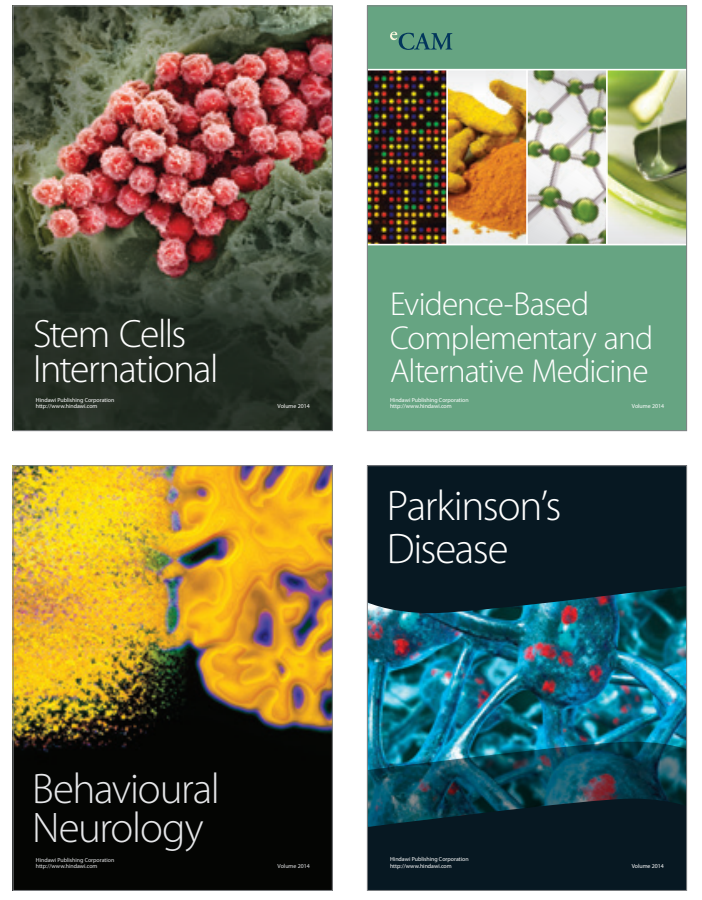
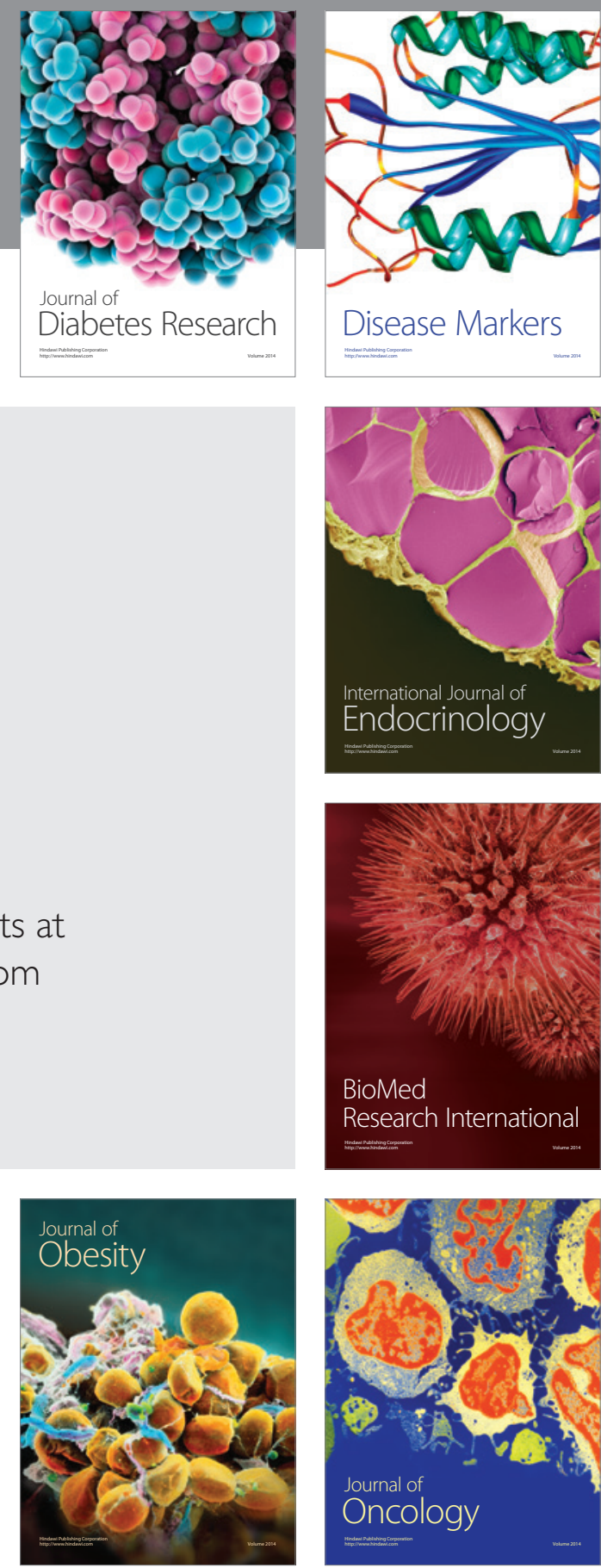

Disease Markers
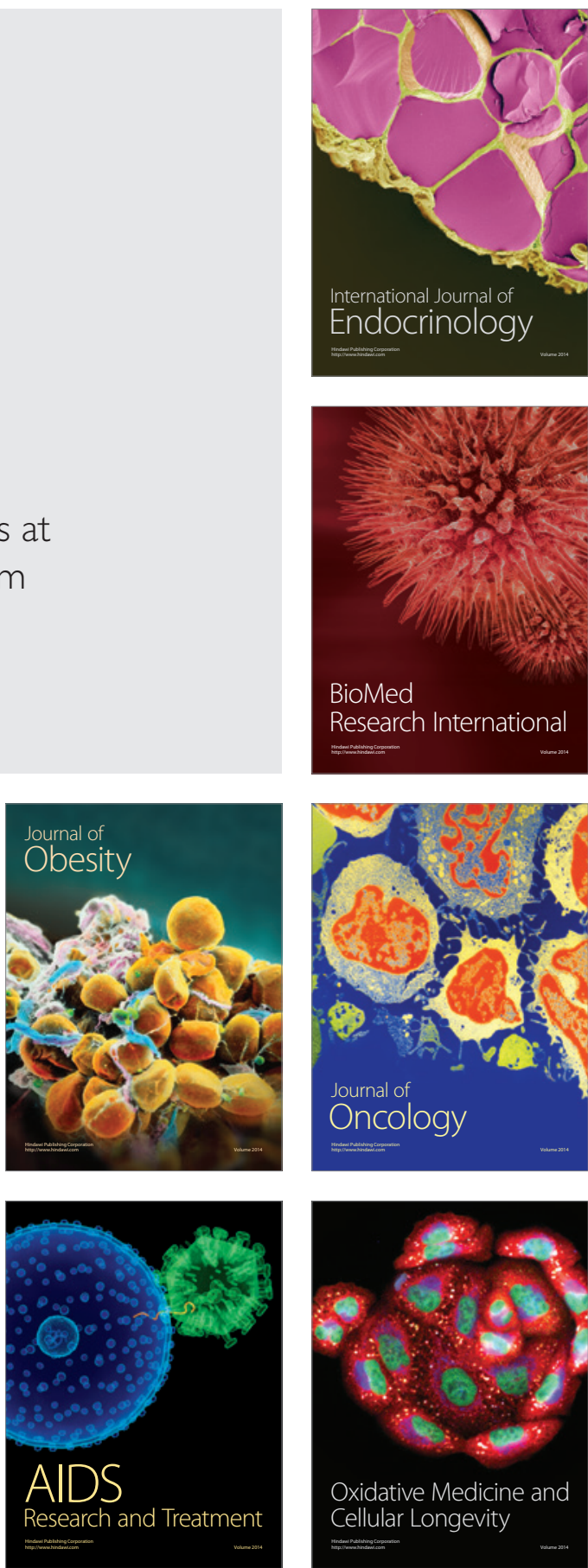